\title{
Improving outcomes following emergency laparotomy: aggregation of marginal gains
}

\author{
MS Liyanage* \\ Consultant Anaesthetist, Mid Essex Hospital Services NHS Trust, Broomfield Hospital, \\ Chelmsford, Essex, United Kingdom \\ NELA Lead for Anaesthetics at Mid Essex Hospital Services NHS Trust
}

\begin{abstract}
Emergency laparotomy is a common general surgical emergency procedure carried out worldwide. It carries with it a very high morbidity and mortality, second only to surgery for ruptured aortic artery aneurysm.

There has been considerable work done to improve the outcomes following emergency laparotomy worldwide. In the United Kingdom, the National Emergency Laparotomy Audit (NELA) was established in 2014. It aims to improve the quality of care for emergency laparotomy patients by providing high quality comparative data collected nationally. It also highlights key standards in the provision of care. The focus of NELA and other quality improvement projects related to emergency laparotomy is improving every step in the patient pathway. This includes access to diagnostic investigations, consultant care, risk stratification and post-operative care in an appropriate destination. Every small improvement in each step would aggregate to provide a considerable reduction in patient mortality and morbidity, as well as reduction in the healthcare burden to the system.

The aim of this review is to discuss some of the significant steps in improving the quality of emergency laparotomy care and the accompanying evidence.
\end{abstract}

Keywords: Emergency laparotomy; outcomes; quality improvement; risk stratification; consultant care

\section{Introduction}

In 2010, Sir David Brailsford, who was appointed General Manager of Team Sky (Great Britain's professional cycling team), faced a tough challenge. Up to that time no British cyclist had won the Tour de France. It was Brailsford's job to make that possible.

He believed in a simple concept - 'aggregation of marginal gains'. Simply it was a $1 \%$ margin for improvement in whatever you would do. If every area was improved by $1 \%$, then they would add up to a remarkable improvement. So, the cycling team looked closely at everything they did including, nutrition, ergonomics, tires, handwashing etc. and optimised every aspect.

\section{*Correspondence: MS Liyanage}

Email: msliyanage@doctors.org.uk

iD https://orcid.org/0000-0002-7050-316X

Received: 15/12/2017

Accepted: 16/12/2017

DOI: http://doi.org/10.4038/slja.v26i1.8304

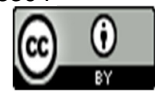

Brailsford believed that by doing this they could win the Tour de France in five years - they won it in three.

Since then, this concept has been adopted in areas other than sport. The doctrine of marginal gains is about small incremental changes in any process, adding up to significant improvement when they are all added together. This concept can be very important when applied to the process of improving emergency laparotomy care.

Emergency laparotomy is one of the commonest emergency general surgical procedures, performed for life threatening abdominal conditions in hospitals all over the world. It describes an exploratory procedure for which the clinical presentation, underlying pathology, anatomical site of surgery and perioperative management vary considerably. ${ }^{1}$ The emergency nature of this operation, the variation in surgical pathology and the limited time available to optimise the patients makes it an extremely high risk operation. Internationally reported mortality rates following emergency laparotomy range from $13-18 \%$ at 30 days. On average one out of every six people who undergo an emergency laparotomy will die within a month of surgery. 
This is second only to short term mortality after emergency open repair of life threatening ruptured abdominal aortic aneurysm. ${ }^{2}$

Recently there has been a lot of initiative to improve the mortality of patients who undergo an emergency laparotomy. It is the focus of several large scale audit and quality improvement programmes including the National Emergency Laparotomy Audit (NELA) in the United Kingdom, the Australian and New Zealand Audit of Surgical Mortality and the American College of Surgeons National Surgical Quality Improvement Program (NSQIP). There is a focus on the patient pathway and making improvements in each step in that pathway so as to aggregate the marginal gains.NELA, in addition to being an audit, also guides quality improvement by emphasising the use of local data to drive small scale changes carried out on short timescales. ${ }^{3}$. There are nine key NELA standards (Table 1)

The aim of this review is to discuss significant factors that may lead to a reduction in the mortality and morbidity of patients undergoing emergency laparotomy, and the recent supporting evidence.

\section{Table 1}

National Emergency Laparotomy Audit (NELA) standards of care ${ }^{4}$

1. CT Scan reported before surgery

2. Risk of death documented preoperatively

3. Arrival in theatre within a timescale appropriate to urgency

4. Preoperative review by a consultant surgeon and consultant anaesthetist when

P- POSSUM risk of death $>/=5 \%$

5. Consultant surgeon and consultant anaesthetist both present in theatre when P- POSSUM risk of death $>/=5 \%$

6. Consultant surgeon present in theatre when P- POSSUM risk of death $>/=5 \%$

7. Consultant anaesthetist present in theatre when P- POSSUM risk of death $>/=5 \%$

8. Admission directly to critical care after surgery when P- POSSUM risk of death $>10 \%$

9. Assessment by a care for the older person specialist for patients aged 70 years and over
Pre-operative risk assessment and scoring systems

Health care resources are not unlimited, even in the developed world. Resource allocation and prioritisation of the sickest patients is a daunting clinical challenge. Therefore, patients need to be risk assessed and triaged in order to place them in the most effective treatment plan.

Risk assessment tools can support clinical judgement in making appropriate treatment plans, informing the patients of the risk of their surgery, determining the post operative destination of the patient and tailoring perioperative care. It also provides an objective support to the clinical diagnosis and could facilitate dialogue between the multidisciplinary teams with regard to patient care.

There are a number of risk assessment tools available for risk stratification ofpatients undergoing emergency laparotomy. In the United Kingdom, the risk assessment system most commonly used for these patients is the $\mathrm{P}$ POSSUM score.

POSSUM stands for Physiological and Operative Severity Score for the enUmeration of Mortality and Morbidity. It was developed by Copeland et al in 1991. ${ }^{5}$ It requires 12 physiological and 6 operative parameters for its calculation (Table 2). However, concerns about over predicting death in the low risk groups led to its modification of the original equation leading to the Portsmouth predictor equation for mortality - the $\mathrm{P}$ POSSUM. ${ }^{6}$

\section{Table 2 \\ P- POSSUM}

$\begin{array}{ll}\begin{array}{l}\text { Physiological } \\ \text { parameters }\end{array} & \begin{array}{l}\text { Operative } \\ \text { Parameters }\end{array} \\ \text { Age } & \begin{array}{l}\text { Operative Severity } \\ \text { Multiple procedures } \\ \text { Cardiac signs }\end{array} \\ \begin{array}{l}\text { Tespiratory history } \\ \text { Systolic blood pressure } \\ \text { Pulse }\end{array} & \begin{array}{l}\text { Peritoneal soiling } \\ \text { Presence of } \\ \text { malignancy } \\ \text { Glasgow Coma Score }\end{array} \\ \text { Haemoglobin } & \text { Mode of surgery } \\ \text { White cell count } & \\ \text { Urea } & \\ \text { Sodium } & \\ \text { Potassium } & \\ \text { Electrocardiogram } & \end{array}$


A P- POSSUM mortality score of more than or equal to $5 \%$ indicates that the patient should be managed by a consultant anaesthetist and consultant surgeon in theatre. A score of more than $10 \%$ indicates that the patient requires critical care admission post operatively. This score and the morbidity score helps in the process of informed consent from the patient and discussion with the patient and family.

However, a systematic review done in 2015, which identified 20 validation studies assessing 25 risk assessment tools in patients undergoing emergency laparotomy, found that APACHE II demonstrated the most consistent outcome. They recommended that the focus should be to recalibrate APACHE II and P- POSSUM in contemporary cohorts, so that they become more accurate. ${ }^{2}$ This may be something which will be of interest in the future.

Researchers have developed a new risk assessment calculator, unveiled in 2017. This uses the NELA database. This NELA risk calculator has been shown to be more accurate than the P-POSSUM scoring system for the UK population, particularly as the risk rises above $15 \% .^{7}$

These scores are now easily available as apps in smart phones and can be used at the bedside of patients to calculate their risk. The use and effectiveness of scoring systems in risk stratification is evidence based. However, it needs to be stressed that they should not be used in isolation. It is an aid to experienced clinical judgement and is not intended to replace it.

More novel methods of risk stratification are the use of bio markers. A recent study has shown that the Neutrophil: Lymphocyte ratio (NLR) of $>8$ is predictive of poorer outcome in patients over 80 years with bowel perforation undergoing emergency laparotomy. ${ }^{8}$ However, more research is needed in this field and it would be interesting to see the direction the use of biomarkers would lead to.

\section{Consultant presence}

Emergency laparotomy is a high risk procedure. In order to make the best possible decision, including the decision to operate in the first place, experienced and senior clinical personnel should be involved early on. The operative procedures involved are often complex and requires a level of expertise and skill and should not be left to the care of an unsupervised trainee. ${ }^{9}$

The Royal College of Surgeons in the United Kingdom, in their document 'Emergency surgery- standards for unscheduled surgical care', have clearly emphasised that in cases with a predicted mortality of $>5 \%$, a consultant surgeon and consultant anaesthetist must be present for the operation. ${ }^{10}$ This has been accepted as a standard of care across the United Kingdom.

The first patient report of the NELA audit published in 2015, placed the risk of dying from an emergency laparotomy in the United Kingdom at $11.7 \%$. Also in this report, $34 \%$ of the patients who undergo surgery are under the direct care of a consultant surgeon and consultant anaesthetist in theatre. ${ }^{11}$ The third patient report of the NELA published this year has reported a reduced mortality rate of $10.6 \%$. This equates to 300 more lives saved per year from 2013. Interestingly 59\% of these patients were under the direct care of consultant surgeons and consultant anaesthetists in theatre. ${ }^{4} 78 \%$ of these patients had a consultant anaesthetist taking care of them in theatre in the third report compared to $43 \%$ in the first. ${ }^{4}$ Although there may be many improvements in practice contributing to the improved mortality demonstrated in NELA, the presence of experienced and skilled clinicians able to make timely decisions and prioritise the sickest patients would definitely be a crucial factor.

In 2017, a study using 23 large hospitals across the United Kingdom, United States and Australia and the Global Comparators Network (GC), looked at the differences in outcome following high risk emergency general surgery admissions in order to determine whether hospital infrastructure factors affect the outcome. This study looked at a large database of 69490 patients. Among other factors the study found that having a consultant surgeon based on site 24 hours a day was associated with a 33\% improvement in mortality rates. Clearing consultant surgeons of elective commitments while on call was associated with a significant improvement $(\mathrm{p}<0.01)$ in the seven-day mortality. ${ }^{12}$

An interesting audit done in the United Kingdom looked at whether increasing the number of on call surgeons to the general surgery rota of a university hospital affected outcomes. This audit 
found that having a second on call consultant surgeon was 2231 times more likely to increase the chances of patients not dying $(\mathrm{p}=0.031){ }^{13}$

It is therefore a persistent recommendation that high risk patients should be managed by the most experienced clinicians. Local protocols need to be examined and developed to facilitate this process.

\section{Goal directed fluid therapy}

Intra operative care of these patients require invasive monitoring, depending on their preexisting co-morbidities, physiological derangement and fraility. The use of fluid resuscitation guided by cardiac output monitoring was recommended in the EPOCH (Enhanced Peri Operative Care for High Risk patients) trial. ${ }^{14}$ Evidence to support goal directed fluid therapy, including a Cochrane review, mainly comes from studies of elective abdominal surgical procedures.

However, these findings may not be generalisable to patients undergoing emergency abdominal surgery. Patients requiring emergency bowel surgery have fundamental pathophysiological differences from elective patients. These include acute inflammation, sepsis, bleeding and fluid disturbances which may be established before surgery even begins. ${ }^{15}$ In addition the use of laparoscopic methods and gas insufflation may also be a factor confusing the accuracy of using dynamic cardiac parameters.

Due to these issues, it seems to be difficult to rely solely on cardiac output guided fluid therapy. However, there are several studies that may answer this issue. The FLO ELA (Fluid Optimisation in Emergency Laparotomy) Trial, which is a multi centre randomised controlled study in the UK, is one example. It ends in 2022. EPOCH trial results may also be able to provide guidance. Until then the most sensible recommendation would be from the International Fluid Optimisation group, who published a statement on perioperative fluid therapy in 2015. Their recommendation was that 'both perioperative fluid choice and therapy be individualised. Patients should receive fluid therapy guided by pre defined physiologic targets. Specifically, fluids should be administered when patients require augmentation of perfusion and are also volume responsive'. ${ }^{16}$

\section{Critical Care admission}

A significant proportion of patients requiring emergency laparotomy, require post operative optimisation and organ support, which could only be provided in a critical care unit. Surgical mortality is influenced by the ability of a hospital to recognise and 'rescue' surgical patients who have developed a complication, and early recognition and management of complications is likely to be better in a critical care unit than on a normal ward. ${ }^{17}$

Critical care resources are limited, even in the developed countries. In comparative studies of the United Kingdom, United States and Australia, it has been found that the mortality rates in the latter two countries are lower. The hospitals included in this study in Australia and USA were found to have a higher ratio of ICU beds per hospital beds and therefore the ability to provide critical care input. A $6 \%$ improvement in mortality was seen for every additional ICU bed per 100 hospital beds. ${ }^{11}$

To illustrate the global nature of this intervention, a large study done in Denmark prior to the era of NELA found that admission to a standard ward before ICU admission was associated with an increased risk of post operative death within 30 days when compared with both ICU admissions after surgery and standard ward care without ICU admissions. The authors identified a number of factors for this including failure to rescue, inability to monitor continuously and less successful prevention of post operative complications due to limited assistance to mobilisation and lung physiotherapy. They concluded that lack of preoperative risk stratification and identification of the high risk patients may be the reason. ${ }^{18}$

Therefore, the use of risk assessment tools and clinical judgement to determine patients requiring post operative critical care and having the dialogue with the intensivists is recommended. This is further supported by several audits done comparing outcomes pre NELA and post NELA. ${ }^{19}$

\section{Pathways and Protocols}

Evidence based practice can be incorporated into pathways and protocols which standardise care to a certain extent. A good and familiar example is the 'Matching Michigan' stepped interventional programme that introduced a bundle of practices 
to minimise central venous catheter (CVC) infections. These technical and non technical interventions brought about a sea change in culture and helped to reduce the mortality and morbidity associated with CVC infections. ${ }^{20}$

Similarly having a pathway for the management of the high risk patient undergoing an emergency laparotomy, providing evidence based steps would help in improving the mortality and morbidity associated with the procedure. ${ }^{17}$ The pathway should incorporate steps that would facilitate timely assessment of the patient clinically as well as by diagnostic investigations, risk stratification, consultant presence in theatre and admission to critical care if required. Best compliance with pathways is when they are locally produced and agreed, with input from key stakeholders in surgery and intensive care. All of these steps combined would lead to a better outcome for the patient as well as to the health system as a whole.

\section{Conclusion}

Improving outcomes in emergency laparotomy, which is common and high risk, requires improvements in all of the steps involved in the process. This process can be facilitated by pathways and working towards accepted standards of practice. National networks sharing data and large databases can guide towards the development of best practice guidelines. As perioperative physicians anaesthetists are best placed to initiate and drive this process along with our surgical and intensive care colleagues. Making sure that these patients are risk stratified, pro active consultant input in the management of high risk patients, individualised fluid management in theatre and critical care admission for high risk patients are key steps in the management which would make a significant difference. Ultimately, small improvements in the patient pathway will translate into large gains and thereby improve patient mortality and morbidity.

\section{Acknowledgements}

The author wishes to thank Drs. P Tilakaratna and $S$. Thanthulage, for their valuable contribution to this review.

\section{References}

1. Nag DS. Assessing the risk: Scoring systems for outcome prediction in emergency laparotomies. BioMedicine. 2015;5(4):20. https://doi.org/10.7603/s40681-015-0020-y PMid:26615537 PMCid:PMC4662940

2. Oliver CM, Walker E, Giannaris S, Grocott MPW, Moonesinghe SR. Risk assessment tools validated for patients undergoing emergency laparotomy: a systematic review, BJA: British Journal of Anaesthesia 2015;115(6): 849860.https://doi.org/10.1093/bja/aev350

3. National Emergency Laparotomy Audit (NELA) - Improving peri operative care. RCoA Bulletin. Issue 93, September 2015.

4. NELA Project Team. Third patient report of the National Emergency Laparotomy Audit. RCoA London, 2017

5. Copeland GP, Jones D, Walters M. POSSUM: A scoring system for surgical audit. Br J Surg, 1991;78: 355-360.

https://doi.org/10.1002/bjs.1800780327

6. Prytherch DR, Whiteley MS, Higgins B et al (1998), POSSUM and Portsmouth POSSUM for predicting mortality. Br J Surg, 1998;85:12171220.https://doi.org/10.1046/j.13652168.1998.00840.x

7. NELA Risk Prediction Tool - RcoA Bulletin, Issue 104, July 2017.

8. Simpson G, Saunders R, Wilson J. et al. The role of the neutrophil: lymphocyte ratio (NLR) and the CRP: albumin ratio (CAR) in predicting mortality following emergency laparotomy in the over 80 age group. Eur J Trauma EmergSurg 2017;Nov 13 https://doi.org/10.1007/s00068-017-0869-4

9. Barrow E, Anderson I, Varley S, et al. Current UK practice in emergency laparotomy. Annals of The Royal College of Surgeons of England. 2013;95(8):599-603.. https://doi.org/10.1308/003588413X1362996004 $\underline{8433}$

10. Emergency Surgery - Standards for unscheduled care, Guidance for providers, commissioners and service planners. Royal College of Surgeons 2011.

11. NELA Project Team. First patient report of the National Emergency Laparotomy Audit. RCoA London, 2015

12. Chana P, Joy M, Casey N, et al. Cohort analysis of outcomes in 69490 emergency general surgical admissions across an international benchmarking collaborative. BMJ Open 2017;7: e014484. doi:10.1136/bmjopen-2016- 014484

13. Hussain A, Mahmood F, Teng C et al. Patient outcome of emergency laparotomy improved with increasing "number of surgeons on call" in a University Hospital: Audit loop. Annals of Medicine and Surgery 2017;23:21-24. https://doi.org/10.1016/j.amsu.2017.09.013 PMid:29021897 PMCid:PMC5633340

14. Enhanced Peri-Operative Care for High-risk patients (EPOCH) Trial: A stepped wedge cluster randomised trial of a quality improvement 
intervention for patients undergoing emergency laparotomy. REC reference 13/EM/0415.

15. Fluid Optimisation in Emergency Laparotomy (FLO-ELA). Open Multicentre, randomised controlled trial of cardiac output guided haemodynamic therapy compared to usual care in patients undergoing emergency bowel surgery. IRAS Project ID 214459.

16. Navarro LHC, Bloomstone, JA, Auler JO et al. Perioperative fluid therapy: a statement from the international Fluid Optimization Group. Perioperative Medicine 2015;4(1):3. https://doi.org/10.1186/s13741-015-0014-Z

17. Saunders DI, Murray D, Pichel AC et al. Variations in mortality after emergency laparotomy: the first report of the UK Emergency Laparotomy Network, BJA: British Journal of Anaesthesia 2012;109(3):368-375 https://doi.org/10.1093/bja/aes165
18. Vester-Andersen M, Lundstrøm LH, Møller MH et al. Mortality and postoperative care pathways after emergency gastrointestinal surgery in 2904 patients: a population-based cohort study, BJA: British Journal of Anaesthesia 2014;112(5):860870. https://doi.org/10.1093/bja/aet487

19. Mak M, Hakeem A, Chitre V. Pre-NELA vs NELA - has anything changed, or is it just an audit exercise? Annals of The Royal College of Surgeons of England. 2016;98(8):554559.https://doi.org/10.1308/rcsann.2016.0248

20. Bion J, Richardson A, Hibbert P, et al 'Matching Michigan': a 2-year stepped interventional programme to minimise central venous catheterblood stream infections in intensive care units in England BMJ QualSaf Published Online First: 20 September 2012. https://doi.org/10.1136/bmjqs-2012-001325 